\title{
Cosmological birefringence induced by neutrino current
}

\author{
C. Q. Geng ${ }^{a, b}$, S. H. Ho ${ }^{a, b}$ and J. N. $\mathrm{Ng}^{b}$ \\ ${ }^{a}$ Department of Physics, National Tsing Hua University, Hsinchu, Taiwan 300 \\ ${ }^{b}$ Theory group, TRIUMF, 4004 Wesbrook Mall, \\ Vancouver, B.C. V6T 2A3, Canada
}

\begin{abstract}
We review our recent work [1] on the cosmological birefringence. We propose a new type of effective interactions in terms of the $C P T$-even dimension-six Chern-Simons-like term to generate the cosmological birefringence. We use the neutrino number asymmetry to induce a non-zero rotation polarization angle in the data of the cosmic microwave background radiation polarization.

PACS numbers: 98.80.Cq, 98.80.Es, 11.30.Fs
\end{abstract}




\section{INTRODUCTION}

The polarization maps of the cosmic microwave background (CMB) have been important tools for probing the epoch of the last scattering directly. As we know, the polarization of the CMB can only be generated by Thomson scattering at the last scattering surface and therefore linearly polarized [2, 3]. When a linearly polarized light travels through the Universe to Earth, the angle of the polarization might be rotated by some localized magnetized plasma of charged particles such as ions and electrons, this is so-called Faraday effect. However, the rotated angle of the polarization plane by this Faraday effect is proportional to the square of the photon wavelength and thus it can be extracted.

On the other hand, in 1997 Nodland and Ralston [4] claimed that they found an additional rotation of synchrotron radiation from the distant radio galaxies and quasars, which is wavelength-independent and thus different from Faraday rotation, referred as the cosmological birefringence. Unfortunately, it has been shown that there is no statistically significant signal present [5, 6]. Nevertheless, this provides a new way to search for new physics in cosmology. Recently, $\mathrm{Ni}$ [7] has pointed out that the change of the rotation angle of the polarization can be constrained at the level of $10^{-1}$ by the data of the Wilkinson Microwave Anisotropy Probe (WMAP) [8] due to the correlation between the polarization and tem-

perature. Feng et al 9] have used the combined data of the WMAP and the 2003 flight of BOOMERANG (B03) [10] for the CMB polarization to further constrain the rotation angle and concluded that a nonzero angle is mildly favored. Recently, Cabella, Natoli and Silk [11], have applied a wavelet based estimator on the WIMAP3 TB and EB data to constrain the cosmological birefringence. They derive a limit of $\Delta \alpha=-2.5 \pm 3.0 \mathrm{deg}$, which is slightly tighter than that in Ref. [9]. For a more general dynamical scalar, this rotation angle is more constrained [12]. If such rotation angle does exist, it clearly indicates an anisotropy of our Universe. This phenomenon can be also used to test the Einstein equivalence principle [13, 14].

In this talk, we will review our recent work on the cosmological birefringence [1]. In Ref. [1], we propose a new type of effective interactions in terms of the CPT-even dimension-six Chern-Simons-like term to generate the cosmological birefringence. In particular, we use the neutrino number asymmetry to induce a non-zero rotation polarization angle in the data of the cosmic microwave background radiation polarization. 


\section{DIMENSION-SIX CHERN-SIMONS-LIKE LAGRANGIAN WITH NEU- TRINO CURRENT}

One of interesting theoretical origins for the birefringence was developed by Carroll, Field and Jackiw (CFJ) [15]. They modified the Maxwell Lagrangian by adding a Chern-Simons term [15]:

$$
\begin{aligned}
\mathcal{L} & =\mathcal{L}_{\mathcal{E} \mathcal{M}}+\mathcal{L}_{\mathcal{C S}} \\
& =-\frac{1}{4} \sqrt{g} F_{\mu \nu} F^{\mu \nu}-\frac{1}{2} \sqrt{g} p_{\mu} A_{\nu} \tilde{F}^{\mu \nu}
\end{aligned}
$$

where $F_{\mu \nu}=\partial_{\mu} A_{\nu}-\partial_{\nu} A_{\mu}$ is the electromagnetic tensor, $\tilde{F}^{\mu \nu} \equiv \frac{1}{2} \epsilon^{\mu \nu \rho \sigma} F_{\rho \sigma}$ is the dual electromagnetic tensor, $\mathrm{g}$ is defined by $\mathrm{g}=-\operatorname{det}\left(g_{\mu \nu}\right)$, and $p_{\nu}$ is a four-vector. Here, to describe a flat, homogeneous and isotropic universe, we use the Robertson-Walker metric

$$
d s^{2}=-d t^{2}+R^{2}(t) d \mathbf{x}^{2}
$$

where $\mathrm{R}$ is the scale factor; and the totally anti-symmetric tensor Livi-Civita tensor $\epsilon^{\mu \nu \rho \sigma}=$ $g^{-1 / 2} e^{\mu \nu \rho \sigma}$ with the normalization of $e^{0123}=+1$.

In the literature [5, 15, 16, 17, 18, 19], $p_{\mu}$ has been taken as a constant vector or the gradient of a scalar. In this paper, we study the possibility that the four-vector $p_{\mu}$ is related to a neutrino current

$$
p_{\mu}=\frac{\beta}{M^{2}} j_{\mu}
$$

with the four-current

$$
j_{\mu}=\bar{\nu} \gamma_{\mu} \nu \equiv\left(j_{\nu}^{0}, \overrightarrow{j_{\nu}}\right)
$$

where $\beta$ is the coupling constant of order unity and $\mathrm{M}$ is an undetermined new physics mass scale. Note that $\overrightarrow{j_{\nu}}$ is the neutrino flux density and $j_{\nu}^{0}$ is the number density difference between neutrinos and anti-neutrinos, given by

$$
j_{\nu}^{0}=\Delta n_{\nu} \equiv n_{\nu}-n_{\bar{\nu}}
$$

where $n_{\nu(\bar{\nu})}$ represents the neutrino (anti-neutrino) number density. It should be noted that if $\Delta n_{\nu}$ in Eq. (5) is nonzero, the cosmological birefringence occurs even in the standard model (SM) of particle interactions [20]. However, the effect is expected to be vanishingly small [20]. In the following discussion, we will ignore this standard model effect. 
As we are working on the usual Robertson-Walker metric, the particle's phase space distribution function is spatially homogeneous and isotropic, i.e. $f\left(p^{\mu}, x^{\mu}\right)$ reduces to $f(|\vec{p}|, t)$ or $f(E, t)[21]$. In other words, the relativistic neutrino background in our Universe is assumed to be homogeneous and isotropic like the $\mathrm{CMB}$ radiation, which implies that the number density for neutrinos is only a function of red-shift z, i.e. the cosmic time. As a result, we conclude that the neutrino current in Eq. (4) to a co-moving observer has the form

$$
j_{\mu}=\left(\Delta n_{\nu}(z(t)), \overrightarrow{0}\right) .
$$

Note that $\vec{j}=-D \vec{\nabla}\left[\Delta n_{\nu}(z(t))\right]$, where D is diffusivity [22] and $\vec{\nabla}$ is the usual differential operators in Cartesian three-space. Here, we have constrained ourselves to consider only the relativistic neutrinos (for homogeneous and isotropic).

As pointed out by Carroll et al [15], in order to preserve the gauge invariance we must require that the variation of $\mathcal{L}_{\mathcal{C S}}$, given by

$$
\mathcal{L}_{\mathcal{C S}}=-\frac{1}{2} \sqrt{g} \frac{\beta}{M^{2}} j_{\mu} A_{\nu} \tilde{F}^{\mu \nu},
$$

vanishes under the gauge transformation of $\Delta A=\partial_{\nu} \chi$ for an arbitrary $\chi$. To check the gauge invariance in our Lagrangian of Eq. (1), we write

$$
\begin{aligned}
\Delta \mathcal{L}_{\mathcal{C S}} & =\frac{1}{4} \chi \tilde{F}^{\mu \nu}\left(\nabla_{\nu} p_{\mu}-\nabla_{\mu} p_{\nu}\right) \\
& =\frac{1}{4} \chi \tilde{F}^{\mu \nu}\left(\partial_{\nu} p_{\mu}-\partial_{\mu} p_{\nu}\right) .
\end{aligned}
$$

From Eq.(6), we have that $\partial_{0} p_{i}=0, \partial_{i} p_{0}=\partial_{i} n_{\nu}(z)=0$ and $\partial_{i} p_{j}=0$, which guarantee $\Delta \mathcal{L}_{\mathcal{C S}}$ in Eq. (8) is zero due to the anti-symmetric property of $\tilde{F}^{\mu \nu}$. Consequently, we obtain that $\tilde{F}^{\mu \nu}\left(\partial_{\nu} p_{\mu}-\partial_{\mu} p_{\nu}\right)=\tilde{F}^{\mu \nu}\left(\nabla_{\nu} p_{\mu}-\nabla_{\mu} p_{\nu}\right)=0$ for the co-moving frame. We remark that Eq. (7) is not formly gauge invariance. In general, to maintain the gauge invariance, we have to introduce the Stückelberg field [1, 23]. Moreover, the existence of a non-zero component $j_{\nu}^{0}$ in Eq. (5) would violate Lorentz invariance [15].

It should be emphasized that the Chern-Simons like term in Eq. (7) is $P$ and $C$ odd but $C P T$ even due to the $C$-odd vector current of $j_{\mu}$ in Eq. (44), whereas the original one in Ref. [15] is $C P T$-odd [24]. It is clear that $\mathcal{L}_{C S}$ in Eq. (7) is a dimension-6 operator and it must be suppressed by two powers of the mass scale $M$. 


\section{COSMOLOGICAL BIREFRINGENCE}

Following Refs. [5, 15], the change in the position angle of the polarization plane $\Delta \alpha$ at redshift $z$ due to our Chern-Simons-like term is given by

$$
\Delta \alpha=\frac{1}{2} \frac{\beta}{M^{2}} \int \Delta n_{\nu}(t) \frac{d t}{R(t)} .
$$

To find out $\Delta \alpha$, we need to know the neutrino asymmetry in our Universe, which is strongly constrained by the BBN abundance of ${ }^{4} \mathrm{He}$. It is known that for a lepton flavor, the asymmetry is given by: [25, 26]

$$
\eta_{\ell}=\frac{n_{\ell}-n_{\bar{\ell}}}{n_{\gamma}}=\frac{1}{12 \zeta(3)}\left(\frac{T_{\ell}}{T_{\gamma}}\right)^{3}\left(\pi^{2} \xi_{\ell}+\xi_{\ell}^{3}\right),
$$

where $n_{i}(i=\ell, \gamma)$ are the $\ell$ flavor lepton and photon number densities, $T_{i}$ are the corresponding temperatures and $\xi_{\ell} \equiv \mu_{\ell} / T_{\ell}$ is the degeneracy parameter.

As shown by Serpico and Raffelt [25], the lepton asymmetry in our Universe resides in neutrinos because of the charge neutrality, while the neutrino number asymmetry depends only on the electron-neutrino degeneracy parameter $\xi_{\nu_{e}}$ since neutrinos reach approximate chemical equilibrium before BBN [27]. From Eq. (10), the neutrino number asymmetry for a lightest and relativistic, say, electron neutrino is then given by [25, 26, 28]:

$$
\eta_{\nu_{e}} \simeq 0.249 \xi_{\nu_{e}}
$$

where we have assumed $\left(T_{\nu_{e}} / T_{\gamma}\right)^{3}=4 / 11$. Note that the current bound on the degeneracy parameter is $-0.046<\xi_{\nu_{e}}<0.072$ for a $2 \sigma$ range of the baryon asymmetry [25, 26]. From Eqs. (15), (10) and (11), we obtain

$$
\Delta n_{\nu} \simeq 0.061 \xi_{\nu_{e}} T_{\gamma}^{3}
$$

where we have used $n_{\gamma}=2 \zeta(3) / \pi^{2} T_{\gamma}^{3}$. For a massless particle, after the decoupling, the evolution of its temperature is given by [21]

$$
T R=T_{D} R_{D}
$$

where $T_{D}$ and $R_{D}$ are the temperature and scale factor at decoupling, respectively. In particular, for $R=1$ at the present time, the photon temperature $T_{\gamma}^{\prime}$ of the red shift $z$ is

$$
T_{\gamma}=\frac{T_{D} R_{D}}{R}=T_{\gamma}^{\prime}(1+z) .
$$


Then, Eq. (9) becomes

$$
\Delta \alpha=\frac{\beta}{M^{2}} 0.030 \xi_{\nu_{e}}\left(T_{\gamma}^{\prime}\right)^{3} \int_{0}^{z_{*}}(1+z)^{3} \frac{d z}{H(z)},
$$

where $H(z)=H_{0}(1+z)^{3 / 2}$ in a flat and matter-dominated Universe and $H_{0}=2.1332 \times$ $10^{-42} h \mathrm{GeV}$ is the Hubble constant with $h \simeq 0.7$ at the present. We note that as the rotation angle in Eq. (15) is mainly generated at the last scattering surface, there is no rotation of the large-scale CMB polarization which is generated by reionization at $z \sim 10$. However, due to the accuracy level of current CMB polarization data, we have assumed a constant rotation angle over all angular scales. Finally, by taking $1+z_{*}=(1+z)_{\text {decoupling }} \simeq 1100$ at the photon decoupling and $T_{\gamma}^{\prime}=2.73 \mathrm{~K}$, we get

$$
\Delta \alpha \simeq 4.2 \times 10^{-2} \beta\left(\frac{\xi_{\nu_{e}}}{0.001}\right)\left(\frac{10 T e V}{M}\right)^{2} .
$$

As an illustration, for example, by taking $\beta \sim 1, M \sim 10 \mathrm{TeV}$ and $\xi_{\nu_{e}} \sim \pm 10^{-3}$, we get $\Delta \alpha \sim \pm 4 \times 10^{-2}$, which could explain the results in Ref. [9]. We note that a sizable $\Delta \alpha$ could be still conceivable even if the neutrino asymmetry is small. In that case, the scale parameter $M$ has to be smaller.

\section{SUMMARY}

In Ref. [1], we have proposed a new type of effective interactions in terms of the CPT-even dimension-six Chern-Simons-like term, which could originate from superstring theory, to generate the cosmological birefringence. To induce a sizable rotation polarization angle in the CMB data, a non-zero neutrino number asymmetry is needed. We remark that

the Planck Surveyor [29] will reach a sensitivity of $\Delta \alpha$ at levels of $10^{-2}-10^{-3}$ [14, 30], while a dedicated future experiment on the cosmic microwave background radiation polarization would reach $10^{-5}-10^{-6} \Delta \alpha$-sensitivity [14].

\section{Acknowledgments}

Two of us (CQG and SHH) would like to thank the organizers: Prof. Manu Paranjape and Prof. Richard MacKenzie for the hospitality during the conference. This work is supported in part by the Natural Science and Engineering Council of Canada and the National Science 
Council of R.O.C. under Contract \#s: NSC-95-2112-M-007-059-MY3.

[1] C. Q. Geng, S. H. Ho and J. N. Ng, JCAP 09, 010 (2007) arXiv:0706.0080 [astro-ph]].

[2] See, for example, W. Hu and M. White, New Astron 2, 323 (1997); A. Kosowsky, Annals Phys 246, 49 (1996).

[3] S. Chandrasekhar, "Radiative Transfer", Chap. 1, Dover, New York, 1960.

[4] B. Nodland and J.P. Ralston, Phys. Rev. Lett. 78, 3043 (1997)

[5] S.M. Carroll and G.B. Field, Phys. Rev. Lett. 79, 2394 (1997).

[6] D. J. Eisenstein and E. F. Bunn, Phys. Rev. Lett. 79, 1957 (1997); B. Nodland and J. P. Ralston, Phys. Rev. Lett. 79, 1958 (1997); J. P. Leahy, arXiv:astro-ph/9704285; B. Nodland and J. P. Ralston, arXiv:astro-ph/9706126.

[7] W. T. Ni, Chin. Phys. Lett. 22, 33 (2005).

[8] C. L. Bennett et al., Astrophys. J. Suppl. 148, 1 (2003); G. Hinshaw et al., Astrophys. J. Suppl. 148, 135 (2003); A. Kogut et al., Astrophys. J. Suppl. 148, 161 (2003); L. Verde et al., Astrophys. J. Suppl. 148, 195 (2003).

[9] B. Feng, M. Li, J.-Q. Xia, X. Chen, and X. Zhang, Phys. Rev. Lett. 96, 221302 (2006).

[10] T. E. Montroy et al., Astrophys. J. 647, 813 (2006); W. C. Jones et al., Astrophys. J. 647, 823 (2006); F. Piacentini et al., Astrophys. J. 647, 833 (2006); see also http://cmb.phys.cwru.edu/boomerang/.

[11] P. Cabella, P. Natoli and J. Silk, arXiv:0705.0810 [astro-ph].

[12] G. C. Liu, S. Lee and K. W. Ng, Phys. Rev. Lett. 97, 161303 (2006)

[13] W. T. Ni, Phys. Rev. Lett. 38, 301 (1977).

[14] For a recent review, see W. T. Ni, Int. J. Mod. Phys. D 14, 901 (2005).

[15] S.M. Carroll, G.B. Field, and R. Jackiw, Phys. Rev. D41, 1231 (1990).

[16] S. M. Carroll and G. B. Field, Phys. Rev. D 43, 3789 (1991).

[17] S. M. Carroll, Phys. Rev. Lett. 81, 3067 (1998).

[18] P. Sikivie, Phys. Lett. B137, 353 (1984); L. Maiani, R. Petronzio, and E. Zavattini, Phys. Lett. B175, 359 (1986); C. Wolf, Phys. Lett. A132, 151 (1988); S. G. Naculich, Nucl. Phys. B296, 837 (1988); A. Manohar, Phys. Lett. B206, 276 (1988); B209, 543(E) (1988); C. Wolf, Phys. Lett. A145, 413 (1990); A. Kostelecky, R. Lehnert, and M. Perry, Phys. Rev. D68, 
123511 (2003); O. Bertolami, R. Lehnert, R. Potting, and A. Ribeiro, Phys. Rev. D69, 083513 (2004); A. A. Andrianov, P. Giacconi, and R. Soldati, J. High Energy Phys. 02, 030 (2002).

[19] M. Li, J. Q. Xia, H. Li and X. Zhang, arXiv:hep-ph/0611192.

[20] J.F. Nieves and P.B. Pal, Phys. Rev. D39, 652 (1989) S. Mohanty, J.F. Nieves and P.B. Pal, Phys. Rev. D58, 093007 (1998);

[21] E.W.Kolb and M.S. Turner, "The Early Universe", Addison-Wesley publishing company, 1990.

[22] See, for example, C. Kittel and H. Kroemer, "Thermal Physics", Chap. 14, W.H. Freeman and Company, 1980.

[23] G. Dvali, R. Jackiw, and S.Y. Pi, Phys. Rev. Lett. 96, 081602 (2006).

[24] S. Coleman and S. L. Glashow, Phys. Lett. B405, 249 (1997).

[25] P. D. Serpico and G.G. Raffelt, Phys. Rev. D71, 127301 (2005).

[26] For recent reviews, see G. Steigman, Int. J. Mod. Phys. E15, 1 (2006); J. Lesgourgues and S. Pastor, Phys. Rept. 429, 307 (2006).

[27] A.D.Dolgov et al., Nucl. Phys. B632, 363 (2002); Y.Y.Y. Wong, Phys. Rev. D66, 025015 (2002); K.N. Abazajian, J.F.Beacom, and N.F.Bell, Phys. Rev. 66, 013008 (2002).

[28] V. Barger, J. P. Kneller, P. Langacker, D. Marfatia and G. Steigman, Phys. Lett. B 569, 123 (2003); J. P. Kneller and G. Steigman, New J. Phys. 6, 117 (2004).

[29] The Planck Consortia, The scientific Case of Planck, ESA Publication Division (2005); see also http://www.rssd.esa.int/index.php?project=PLANCK.

[30] A. Lue, L. M. Wang and M. Kamionkowski, Phys. Rev. Lett. 83, 1506 (1999). 